\title{
GIS for Environmental Problem Solving
}

\author{
Koushen Douglas Loh and Sasathorn Tapaneeyakul
}

Additional information is available at the end of the chapter

http://dx.doi.org/10.5772/50098

\section{Introduction}

The authors are affiliated with the Laboratory of Systems Technology Applications in Renewable Resources (The STARR LAB) at Texas A\&M University. The purpose of this chapter is to provide a synopsis of the cumulative research and teaching work for the past twenty years from the STARR LAB. The aim of chapter is to demonstrate holistic understandings of what key environmental issues and problems people are facing and how their concerns may be addressed with the help of geographic information systems (GIS).

We are the environments, and the environments are us. There are many environmental issues and problems the society is facing. Some major categories include environmental disasters, ecological services, and perceptions of environments by people, just to name a few. In terms of environmental disasters, hurricanes, earthquake and wildfires are some examples that exert enormous direct impacts on people's lives. Their increasing recurrences have elevated public awareness on the vulnerability and risks of the environments we live in. An awareness of environmental issues leads to an increase in people's perceptions regarding the surrounding environments. There are many factors contributing to such perceptions. Combined considerations of pertinent factors result in an overall perception. One plausible combined index is called quality of life (QOL). QOL is a practical measurement of the state of an environment. Environmental awareness also raises people's concerns on the sustainability of the ecological services. Ecological services refer to public goods, tangible or intangible, rendered to us by environments and ecosystems. Air, water, food, fiber, and fuel we consume are good examples. Sustaining these services is of great importance to all environmental stakeholders.

There are many ways to help stakeholders gain insights to environmental issues and problems. One handy approach is the use of GIS. GIS are systems of hardware, software, data, people, organizations and institutional arrangements for collecting, storing, analyzing and disseminating information about areas of the Earth [1]. Such technologies enable 
analyses of spatial-temporal patterns for a geographic span of interest and generations of easy-to-comprehend reports such as maps and images. GIS are maturing and proliferating rapidly in parallel to the quantum leap of personal computer (PC) platforms. It greatly enhances people's ability to know about their environments. Given the advantages, GIS have emerged as a popular subject matter among interested learners on college campuses as well as in environmental fields. A good indicator of this assertion is the sustaining popularity of Environmental GIS courses the authors teach at Texas A\&M University. Other institutions are reporting a similar phenomenon.

All things considered, it is timely to provide a rundown of GIS for Environmental ProblemSolving as a chapter of this book. Main thrusts of our presentation consist of four parts. They are: 1) Introduction (this section); 2) Research method; 3) Illustrations of GIS for environmental problem solving applications; and 4) Concluding remarks.

\section{Research method}

Systems approach is a key research method to incorporate GIS into problem-solving process in addressing environmental issues and problems. The essence of this approach is to envision and to enact relevant endeavors into a cohesive sequence of steps. The whole process is called developing and implementing a GIS project. A typical sequence of steps in a GIS project includes framing the problem, defining a project area, identifying and acquiring data, extracting and preparing data, editing spatial data, geospatial analysis, and generating maps and reports.

\subsection{Framing the problem}

The first step in solving any problem is to frame the problem. The purpose of this step is to help narrow down the scope and identify the problem to make it easier to solve. This helps address the questions you want to answer. Specifically, what do you want to accomplish from looking at this problem? What are the goal and objectives you are planning to address from the problem?

Then, the next question is what is the potential information associated with the problem? Pertinent information includes:

- Scope: To lay out tasks, data, and time frame to solve a problem, a scope needs to be defined so that you know how much information you are dealing with. The scope varies depending upon the nature and objectives of the problem. Questions on whether the problem is looking at a specific region, a particular group of population, or a particular phenomenon are worth investigating. Also, is the problem asking for information, maps, or more in-depth analysis of the problem?

- Scale: Is the problem focusing on an institutional scale (individual, family, municipal, state, national, or international) and/or ecological scale (plant, plot, ecosystem, landscape, biome, or global)? As addressed in [2], stakeholders at different spatial scales can (and should) assign different values to environment and ecosystem under interest. 
- Type of information: two distinctive types of information are quantitative and qualitative. You need to specify if the problem is looking for quantitative and/or qualitative information. Quantitative information focuses on some sort of value or measurable information. Number of population affected by a hurricane or the amount of oil spilled into an ocean are quantifiable. Qualitative information, on the other hand, represents some sort of status that needs to be stated. Wildlife species affected by a hurricane or types of chemical released into a river are some of the examples.

It is also helpful to construct an outline or diagram of the problem so that it is easy for you and/or stakeholders to determine necessary steps, to better organize the tasks, and to be able to comprehend the problem at hand.

Consider the following real world examples using the above criteria:

\section{Example 1: The 2005 Hurricane Katrina}

The scope is the Hurricane Katrina in New Orleans, Louisiana. This pertains to the Greater New Orleans Region. Information of interest includes population affected, infrastructural damage, hazardous materials, and situations that might arise afterward. Given this information, one possible answer is the number of population affected as the quantitative information. Quantitative information includes, but not limited to, current stage of hazardous waste, groups of population, animal species, and housing.

\section{Example 2: Bastrop County Complex Fire}

The scope is a major wildfire in Bastrop County, Texas in 2011. Information of interest is effects on both human and animal, economics losses, effects on land and environments, and infrastructural damage. With the defined information, possible answers include the number of affected people and animals, income losses from the incident, and the loss of species' habitats, which are accounted as quantitative information. Households and habitats affected by the fire, problem of land degradation and fragmentation, time frame for recovering, and preventive plans are some of the qualitative information that seeks answers.

\section{Example 3: West Nile Virus in Brazos County}

Brazos County, Texas and the surrounding areas is the scope of interest. Became widespread in the recent years (with the highest number of 7 severe cases in human being accounted for in 2006 [3]), West Nile virus has been under surveillance for residents in the County. Critical information that needs to be asked include: What causes the West Nile virus?; How can you track the spread of the West Nile virus?; and Where has West Nile virus been found in this location? Quantitative answers are the current number of infected individuals and the past records. Possible locations and trends that may be associated with the spread of the West Nile virus serve as the qualitative answer to the problem.

\subsection{Defining a project area}

With an identified problem, you can proceed to define a project area. This step delineates a confined boundary of an area of interest. The information from Step 2.1 helps specify the 
proper location where the problem occurred and address the possible questions and answers under interest. The process pinpoints the focus of the problem while eliminate unnecessary areas or secondary scope of interest from the picture. Not only that this can help save time, but it also allows you to pay closer attention to the essence of the project. At this stage, the conceptual project area should be carefully thought out before attempting to acquire data, i.e., map layers, in the next step.

GIS enable a variety of ways for convenient delineation of a project's boundary that might not be made possible with other applications. ArcGIS ${ }^{\circledR 1}$, a worldwide used GIS software, allows users to work with geographic information data by inputting and manipulating map layers in a comprehensive manner. In this chapter, we use ArcGIS 9.3.1 for all GIS applications.

For example, one may select an administrative boundary of a local jurisdiction from a base map layer as the project area as in the cases of Bastrop County Complex Fire and West Nile Virus in Brazos County. One may also "union" multiple local jurisdictions into a broader geographic span for addressing issues that are of cross-boundary nature. The project boundary resulted from one way or another serves as the "cookie cutter" for clipping data from relevant layers and tables in the ensuing steps to expedite problem-solving. As in the case of Hurricane Katrina, at least five parishes (Louisiana's equivalent of counties in other states) should be included as the project area of the Hurricane analysis.

\subsection{Identifying and acquiring data}

Once the project area is defined, the next step is to locate and acquire needed data. Before looking for data, the methodology needs to be analyzed to establish what data is needed. The most important question that needs to be answered is: Why do I need this data? If the data is truly needed, then this question is easily answered. If not, then the data is most likely not necessary to solve the problem.

To be able to work with data in GIS, you need to understand the nature and procedural steps of working with data in GIS as follows:

\subsubsection{GIS datasets formats}

Typical formats of datasets, which allow you to conveniently work with multiple information or map layers, include spatial and attribute data.

Spatial data comes in the forms of raster and vector and is generally organized into so-called layers or thematic maps.

- Raster data is digital image composed by rectangular grids or cells that contain numeric information from a defined range to characterize geographic features. Digital Elevation Model or DEM is a form of raster data important in depicting a terrain. It provides crucial information on the topologies of a geographic span.

\footnotetext{
${ }^{1}$ ArcGIS is a registered trade mark of Environmental System Research Institute (ESRI), Inc.
} 
- Vector or shapefile data is constructed as points, lines, and polygons to represent geographical features.

Attribute data is information used to describe characteristics of a locale. The data is organized in a table containing information linked to a spatial feature by a common identifier. This gives you details or certain types of information associated with each specific feature.

\subsubsection{GIS data sources}

GIS data is vastly available from many sources, including those in public domains at local, state and federal agencies; international non-governmental organizations or NGOs; and private sector providers. In the U.S., the Federal Government generates and provides public access for easily found and downloadable geographic datasets ${ }^{2}$. Each agency supplies relevant datasets pertaining to their line of work. Some of the main providers include: National Oceanic and Atmospheric Administration (NOAA) for extensive National Weather Service datasets; United States Geological Survey (USGS) for DEMs, administrative boundaries, land cover, soil, and water data; United States Environmental Protection Agency (EPA) for toxic and cleanup sites; and Census Bureau for demographic and socioeconomic data, which contains a widerange of attributes detailed to the block level. State agencies, such as Texas ${ }^{3}$, have established a central clearinghouse for state-level datasets. With increasing demand for GIS in solving various problems, many counties and cities have initiated GIS departments, which oversee and provide relevant geographic data to inquirers.

\subsubsection{Map projections and coordinate systems}

Each map layer contains a coordinate system, which allows one to identify the location of the map and to be able to display, manipulate, and integrate the map layer with other layers for further applications and analysis. It is therefore imperative to understand the fundamentals of map projections and coordinate systems.

A coordinate system is a grid that may be used to define where a particular location is. Two common types of coordinate system are:

- Geographic Coordinate System: This uses 3D spherical surface to define locations. Often incorrectly referred to as datum, geographic coordinate system includes not only datum, but also angular unit of measure and prime meridian. Points on Earth's surface are referenced by latitude and longitude, while angles are measured by degree.

- Projected Coordinate System: Commonly referred to as map projections, projected coordinate system is defined on flat, 2D surface with constant lengths, angles, and area. $\mathrm{X}, \mathrm{Y}$ coordinates are presented on grid. It is based on geographic coordinate system.

Often, input maps will be in different projections, requiring transformation of one or all maps to make coordinates compatible. Since monitor screens are analogous to a flat sheet of paper, there is a need to provide transformations from the curved surface to the plane for displaying

\footnotetext{
${ }^{2}$ See http://geo.data.gov/geoportal/ for more information.

${ }^{3}$ See http://www.tnris.org/get-data/ for more information.
} 
data. In order to do so, mathematical formulas to relate spherical coordinates to planar coordinates are required. Some distortions in the shape, area, distance or direction of data can occur during the transformation; different projections cause different distortions. Therefore, careful consideration of the appropriate map projection is crucial. Proper map projection must consider: the map's subject and purpose; the subject area's size, shape, and location; the audience and general attractiveness; size and shape of page; and appearance of the graticule ${ }^{4}$.

\subsection{Extracting and manipulating data}

The fourth step is data extraction and manipulation. In this step, one is to extract data from a conceivably larger original source file. Reduction of the size of datasets and their consolidation expedite the ensuing data management and processing. The project area defined at the onset (the cookie cutter) dictates the extent and size of data to be extracted and prepared.

Typically, data acquired may exist in various forms and shapes, e.g. different coordinate systems and file formats. It is a MUST to prepare and consolidate all datasets into a commonly operable format. GIS have a database management system component to support the proper management of both spatial and attribute data. It also enables convenient linking and relating of various data records by their locations on a common coordinate system. Some common tasks you will encounter during the data extraction and manipulation steps are as follows:

- Re-projecting data: This is a basic essential step in any analysis using GIS. The purpose is to convert a particular piece of data from one coordinate system to another. Working with GIS employs more than one map layer, therefore acquired datasets may contain different projections. Different data projections lead to distortion of data and inaccuracy in the analysis.

For example, in Figure 1, a residential area polygon (in blue) is projected to Geographic Coordinate System: GCS_North_American_1983. The same residential area polygon (in yellow) is in Projected Coordinate System: NAD_1927_UTM_Zone_16N. As shown in Figure, there are some discrepancies in the map layers with different coordinate systems. If this re-projection step is not taken, any analysis preformed will be inaccurate leading to much larger problem in subsequent analysis with multiple layers.

- Conversion of raster to vector: Not only data comes in different coordinate systems, the file formats can also be varied; most commonly in the forms of raster or vector (shapefile). Especially with the growing use of GIS, datasets in shapefile have become more available. Shapefile data usually comes embedded with attribute data, which allows user to easily select and manipulate the information of interest. Therefore, converting a raster file to vector enables user to intersect other data with the available vector data. Suppose you have acquired and managed shapefile layers of affected area by Hurricane Katrina and population layer in the Greater New Orleans Region, by intersecting these two layers, you can extract the areas in which population were affected by the Hurricane.

${ }^{4}$ See [4] for further explanations on coordinate systems 
- Reclassification: To extract specific data from a raster, i.e., specific elevation data, reclassification is performed. Given the Hurricane problem, flooding can be assessed as one major result of the incident. In order to extract only the flooded area resulted from the Hurricane, reclassification is utilized to distinguish a specific range of elevation in which flooding occurred from others. This will allow you to analyze the effects pertaining to the flooded area.

- $\quad$ Selecting by attributes: The purpose is to extract desired attribute data for analysis. This can be done through conditional statement imposed in attribute data table to select only specific information of interest. Considering an attribute table of chemical sites located within the Hurricane flooding zone, one can select only specific sites containing particular chemicals of interest for further analysis and map report.

- Exporting data: To make a temporary layer permanent in a current map, data resulted from steps such as that of above need to be exported and saved in a current working folder. Otherwise, the file may be lost or difficult to locate when you want to revisit and work on it.

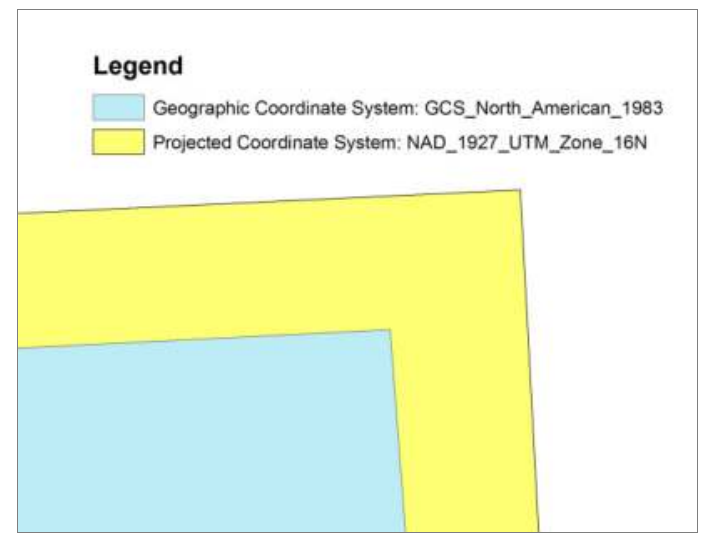

Figure 1. Same layer file with different coordinate systems resulting in 20-meter difference on the map.

\subsection{Editing spatial data}

Oftentimes, acquired data might not be in the most suitable shape or boundary for problem under consideration. Options to edit spatial data in GIS allow one to manage the data in such a way that is more manageable and ready to be analyzed.

Typical editing tools consist of creating new features, cutting polygons, modifying features, and extending the basic skills to other tasks such as clipping a feature to a desired shape and area.

- Creating new features: When creating a new feature, a blank data set is being defined by the editor. A blank data set is like an empty pie shell, while creating a new feature is like filling the pie shell. This task is only used if a new feature is desired or a single part feature is to be converted into a multi-part feature when the second part of the feature does not already exist. 
- Cutting polygon features: This process is a shortcut to creating a multi-part feature from a single part feature. Simply put, this process is used like a set of scissors to cut an existing feature into multiple parts.

- Modifying features: This task is used when an existing feature does not cover the area that is desired. The attribute data will remain the same, while the feature will be modified to suit one's need.

- Clipping features: Clipping is a process that is like using a "cookie cutter" to remove a portion of a feature permanently. The attribute data will also be changed due to a permanent removal of the feature.

\subsection{Geospatial analysis}

Upon data readiness, a project may move on to the sixth step of spatial-temporal analyses. There are many useful procedures for these endeavors. Especially with the versatilities of GIS software, one can utilize extended range of applications available. Some common tools that one should be familiar with and were used specifically for the ensuing applications in this chapter include:

- Distance analysis: A suite of tools to produce distance maps are commonly available in GIS. In ArcGIS, distance tools are available under Spatial Analyst option. Euclidean distance tool measures straight-line distance from the center of cell to the nearest object of interest, i.e., your source. Another alternative is the cost distance tool, which incorporates travel cost from different paths into the analysis. The products from these tools are distance maps in raster representing proximity maps with a range of distance values from the source. For instance, one can find proximities from pollution sources at defined interval to any locales within a defined area map.

- Map algebra: Another useful application, which you will encounter at certain point of analysis, is map algebra. This can be used for computations of raster data to create spatial patterns that depict locales of a particular concern or interest. Raster calculator, a Spatial Analyst application, allows for this useful procedure by inputting specified mathematical functions and expressions in the calculator. The result will be raster values and layer corresponding to the specified function.

The use of analytic procedures mentioned above and other tools in a proper order results in useful information for a problem under study.

\subsection{Generating maps and reports}

The final major step is to generate maps and reports. One picture is better than a thousand words. To this end, GIS come handy in presenting information in maps, images, 3D graphs, tables, and other forms. It also expedites the import and export of these presentations between GIS and other software environments, e.g. a word or a graphic processor. With the acceleration of PC powers, the sky is the limit to GIS' capability of generating maps and reports. It is worth noting that you should understand what the readers are looking for when creating the maps and write ups, i.e., what is the focus or message that you want to communicate to others? This should align with the proposed information of interest. 


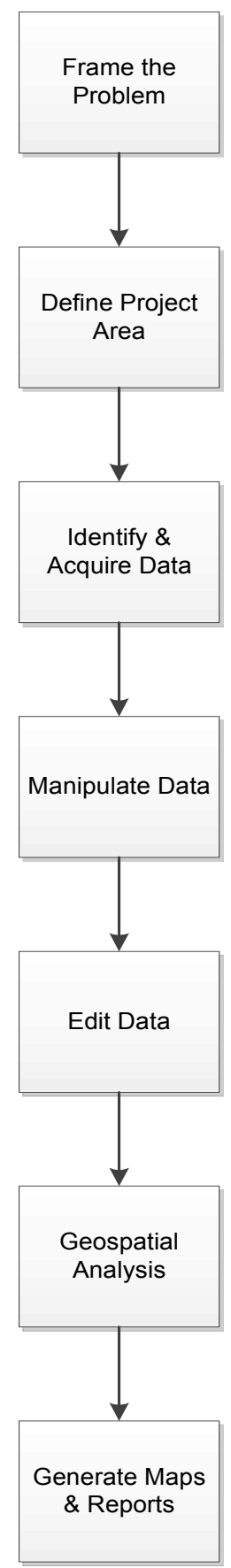

Figure 2. Diagram of problem-solving steps 


\section{Illustration of GIS for environmental problem solving applications}

To illustrate how GIS are used to help address environmental issues and problems, two cases are described herewith in this section. The first one is on flood assessment, and the second is a QOL analysis. The applications help prepare for the building framework of spatial appraisal and valuation of environment and ecosystems (SAVEE), which will be discussed in the following section, tremendously.

\subsection{Flood assessment}

Considered one of the costliest [5] and most destructive natural disasters in the history of the United States, Hurricane Katrina provides a number of opportunity to understand the risk of nature, and how one could expect to understand and learn from such disastrous effects. The aforementioned problem-solving steps allow us to contemplate the steps as follows:

Step 1: Framing the problem

An analysis of the scenario indicated that Hurricane Katrina occurred in the Greater New Orleans Area. Field measurements and distributions on the majority of victims indicated that roughly those under 1 meter in elevation were initially affected by the flooding [6]. Given this information, the scenario was that every location below 1 meter in elevation was affected and any location that is above this level was unaffected by the flood water. This particular area of impact needed to be delineated. The information of interest included area and population affected by the Hurricane. Additional scenarios of water-rise were then set for 5, 10, and 15 meters to emulate different levels of flooding.

\section{Step 2: Defining the project area}

In this case, the City of New Orleans and its five neighboring parishes suffered by the storm were identified as the study area.

\section{Step 3: Identifying and acquiring data}

The best type of data for delineating the affected area is the elevation data (DEM). DEMs, Satellite Imagery, and Census datasets were collected from Atlas, the GIS data central from the State of Louisiana [7]. This included DEM, jurisdiction boundaries, street maps of the study area, and Census data.

\section{Step 4: Extracting and preparing data}

Initially, the DEM and Census data came projected as GCS_North_American_1983. By assigning a projected coordinate system to the data, further analysis could be proceeded. Given the information, we projected the data to NAD_1927_UTM_Zone_16N. Hillshades of the DEMs were also generated to visually inspect different elevations in the data. Sink holes pervaded in the DEMs were also been filled to prevent erroneous and prepare for proper flow direction process.

Then, the second crucial step was to extract the flooded area from the total area. This employed the reclassification process in which the elevation value was changed to 1 meter to separate the 
flooded area from the non-flooded area (elevation above 1 meter). In short, the reclassification divided the elevation data into the flooded area and the non-flooded area. The rest of the waterrise scenarios then followed using the same reclassification step as well as the ensuing steps.

Next, this flooded elevation data was converted into vector to prepare for further analysis. The converted flooded layer was the result of the conversion process as well as exporting the data into a new permanent flooded area layer.

In terms of the Census data, the parishes were merged into one layer so that it was more convenient to work with in the subsequent steps for analyzing the total effect on population.

\section{Step 5: Editing spatial data}

The acquired data contained certain parts that were irrelevant to the analysis. Lake Ponchartran, for instance, should not be counted toward the flooded area. Therefore, by editing the data, some unnecessary information of interest were taken out. Pertinent steps of editing the lake included: 1) Creating a new blank shapefile; 2) Using the blank shapefile as the base for editing tools to create a new feature around the lake area; and 3) Using the newly created feature as a cookie cutter to clip off the lake area from the flooded area layer. The result was the flooded area without the lake that was ready to be incorporated into other analysis.

\section{Step 6: Geospatial analysis}

At this stage, socio-economic analyses were conducted to assess the damage and impact on the livelihoods of residents of the affected areas. Census data developed was used directly for this purpose. Combining census data with the emulated flooded areas, patterns of suffering by which racial stakeholders and by what economic classes were clearly displayed.

Based on the flooded area layer in Step 5, we proceeded to calculate the area under the layer's attribute table. Visual Basic Code to calculate the area (available from [8]) or a Calculate Geometry option, an automated tool in ArcGIS, derived the numbers of area affected by the Hurricane. Mathematical formula imposed helped convert the numbers into desirable units such as acres.

Benefited from the above numbers, the population affected was conveniently calculated. Census data contains racial information that represents groups of population in different parishes. By intersecting the flooded area layer with the merged parishes layer, representing population profile in the areas, affected population was extracted. The overall statistics in the attribute table identified the total population affected by the flooding. Figure 3 demonstrates map layers resulting from the above problem-solving steps.

Step 7: Generating maps and report

Upon generating desired information and analysis, each pertinent map was composed as a map report containing a map title, legend (showing values of the map layer), north arrow, and scale bar. Then, the map reports were exported as image files to be included in a report. The report addressed the finding results of effects from the Hurricane as illustrated by the maps and relevant discussions of further applications and analysis that can later be applied based on this project. 


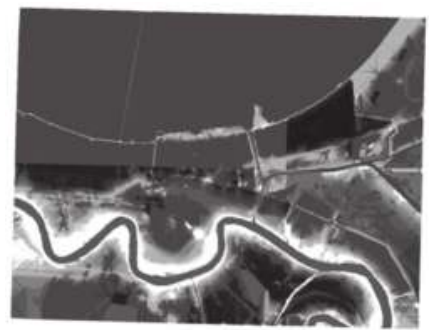

Merged DEM layers of project area with sinks filled

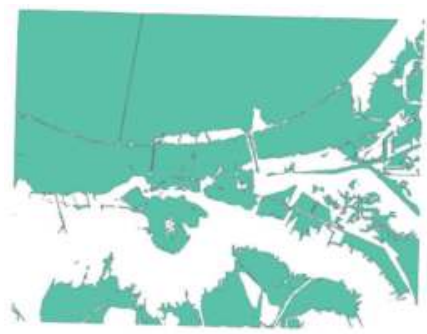

Flooded area layer with lake portion

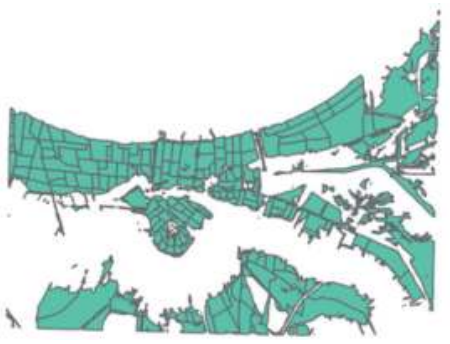

Intersection of Census data with flooded area

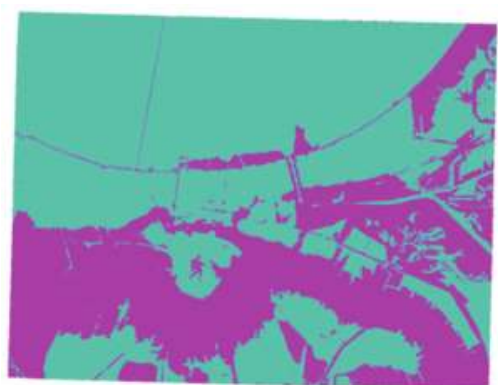

Legend

Flooded Area

Non-flooded Area

Reclassification distinguishing flooded area (in blue) and non-flooded area (in pink) at 0 meter water

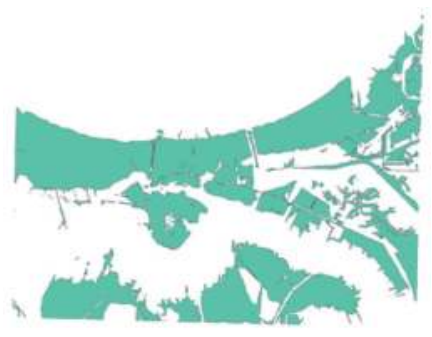

Flooded area layer without lake portion

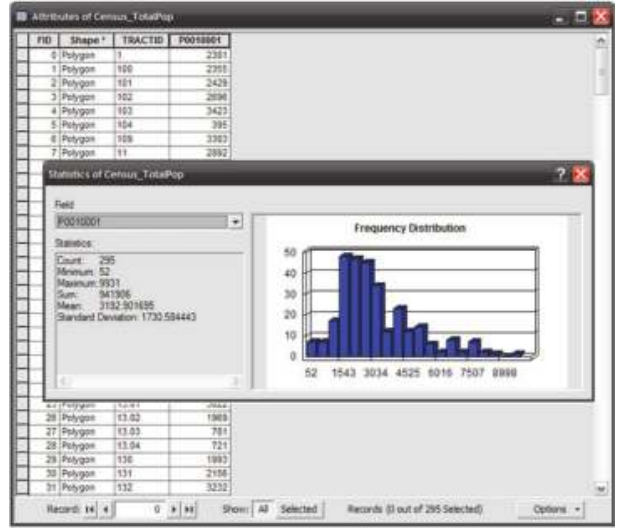

Attribute table and statistics of total population affected

Figure 3. Illustrations of selected problem-solving steps for flood assessment in reference to the 2005 Hurricane Katrina flooding in the Greater New Orleans Region. 


\subsection{Quality of life assessment}

QOL is emerging as a major indicator to monitor citizen's livelihood and wellbeing at the grassroots level. By virtue of its focuses, QOL helps inform local people and organizations of their living environment and optimize the allocations of resources to improve the community development. Canada is perhaps more aggressive in setting up a national framework for QOL [9]. In the U.S., states such as Utah [10]; cities such as San Francisco, California [11]; and organizations, including nonprofit organizations such as the Quality of Life Foundation [12] have been vigorously promoting such term as one of their agendas.

Categories of data to support the development of QOL indicators range from education, environment, economics, social, and justice to transportation/mobility. However, the use of GIS to track QOL progress is still at its infancy stage. City of College Station, Texas, with its advanced GIS installation and rich collection of data, stands to gain a lead role in this area and to provide even superior services to its residents when it embarks on this path.

There are three issues and opportunities in the development of QOL indicators. They are:

- Combining subjective values with objective measurements to create consensus and develop common ground to accommodate multiple perspectives of stakeholders.

- Combining the use of both spatial and attribute information to develop base layer and indices in environment, crimes, recreation, etc. For example:

- Overlay of census blocks with subdivisions or other neighborhood entities (e.g. apartment complex) to establish the baseline reference (population, its composition, income level, education level, and number of household of an entity)

- Overlay of crime type, frequency, and location data with entities on the base layer

- Developing a composite score (ranking) of QOL for each neighborhood entity on the base layer

As you set forth to do your research, as in the case of QOL assessment, you are most likely facing with three puzzling situations:

1. Pertinent data/information comes in a variety of forms

It is plausible that the data/information you are facing and plan to collect exists in at least two forms. They are categorical and numeric. Examples of categorical information include "Yes" or "No" on whether a city (or any local jurisdiction) has a neighborhood improvement in place or not program; "Very Good," "Good," "Fair," and "Bad" on how such a program is being regarded by the communities; and "Highly favorable," "Favorable," and "Least Favorable" on how service rendered by the program is perceived by the beneficiaries.

Quite often, information of categorical nature is derived from one's "gut feeling." It may also be convenient to summarize some judgments based on historical data, on some kinds of trends, or on some opinion surveys/polls.

There are two types of numeric information: discrete and continuous. Population of an ethnic group residing in a particular Census unit is an example of discrete type. Example of continuous type is the percentage of an ethnic group versus the total population in such a unit. 
2. How to "add" "oranges" and "apples"

When one has data and information of various types in hand, he/she will ask this question:

"How do I add them together?" Indeed, you cannot add oranges and apples together at their original forms. The trick is to convert and normalize all of them into the same numerical scale, say between -1 and +1 .

So, what is normalization? Normalization is the act of taking many sets of data that have no clear correlation and placing them under the same quantitative scale. Essentially, normalization allows us to compare apples and oranges. Some decisions must be made prior to normalizing any type of data. The questions include:

1. What are the important factors?

2. Which factors are positive and which factors are negative?

3. How much should each of these factors count in relation to the overall project?

For categorical type of information, what you do is to fix the "best" and the "worst" at +1 and -1 respectively. This is plausible as +1 can represent the best case and -1 the worst. When both ends are fixed, one may logically deduce that a numeric value of " 0 " represents "Inconclusive." Furthermore, one may come up with a scheme saying that " +0.25 " is "somewhat better", " +0.5 " is "better" and " +0.75 " is "much better." One can also say that "0.25 " is "somewhat worse", "- 0.5 " is "worse", and "- 0.75 " is "much worse." As a result, you are converting and normalizing categorical or qualitative data into numeric or quantitative information as illustrated in Figure 4.

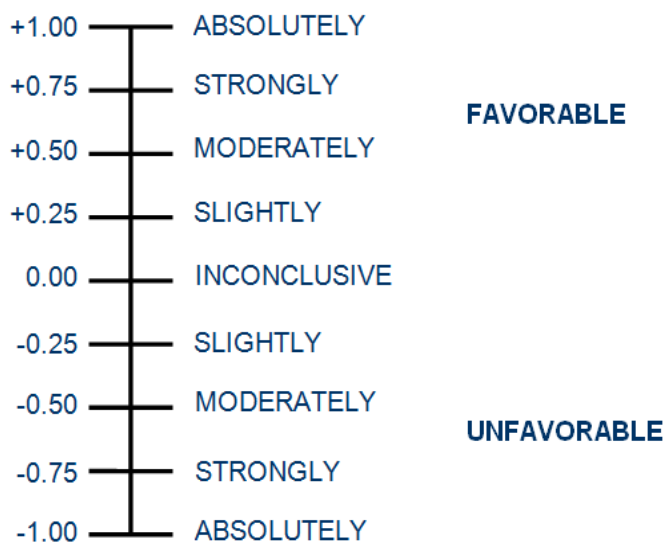

Figure 4. Illustration of the qualitative - quantitative information conversion scheme. 
For numeric information, the conversion and normalization is less complicated. Say you deem the ratio of white population in a Census unit at $50 \%$ is the best mix (most favorable), in terms of quality of life; $100 \%$ or $0 \%$ is least favorable. For the best mix, you believe it should be given a score of +1 and for the least favorable a 0 . Given this range, you may apply the following equations to convert and normalize the percentage into values in the range $(0,+1)$

$$
F(x)=\left\{\begin{array}{l}
1-\frac{(x-E)^{2}}{(1-E)^{2}}, E \leq x \leq 1 \\
1-\frac{(x-E)^{2}}{E^{2}}, 0 \leq x<E
\end{array}\right.
$$

where $\mathrm{E}$ is the expected (best value of $\mathrm{x}$ )

Another method is to convert distance to an object from such measurements as miles to the uniform score between $(-1$ and +1$)$. For example, one may decide that the presence of oil well is bad for quality of life. Evidently, the household right at the oil well would have absolutely unfavorable score of -1 . The negative effect most likely would tap off as the distance reaches certain threshold, e.g. 1 mile or 5,280 feet. The tapering effect can then be described by a negative exponential equation as:

$$
F(x)=-e^{-x}
$$

where $\mathrm{x}$ is the distance to oil well(s)

The normalization equation for strictly negative attribute based on the negative exponential equation above becomes:

$$
Q O L_{i}=\operatorname{Con}\left(\left[\text { Distance }_{i}\right]<X, e^{-\left(\frac{\left[\text { Distance }_{i}\right]+1}{X}\right)}, 0\right)
$$

The translation of the equation is "If Condition $<X$, True, False." This means if an input value (distance value) falls under the condition (less than $X$ ), then the output is negative value. Otherwise, the output is zero. It might be helpful to put this in the oil well scenario above:

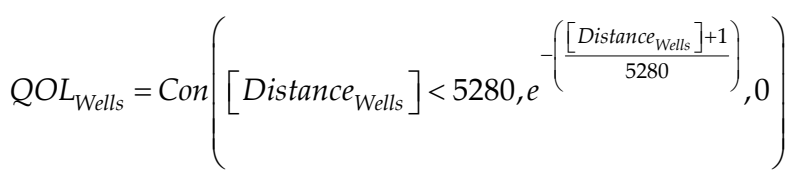

The above equation is set so that if the distance to an oil well is less than 1 mile, then the output is negative. As the locales get closer to the actual oil wells path, the more negative they will become (with the minimum at -1). At a distance of 1 mile or greater all the output values are set to zero as shown in Figure 5. 


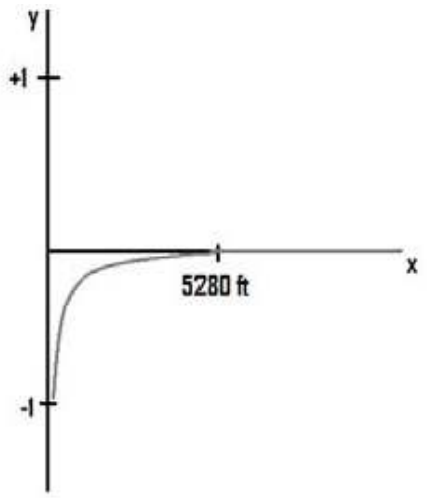

Figure 5. Negative decay graph showing the more negative values as the locales get closer to oil wells.

At any rate, once you have all factors converted and normalized into the scheme of $(-1,+1)$, then the values can be "operated" on to add up their contributions to the overall quality of life assessment of a city. This is done by applying the following formula:

$$
\begin{aligned}
& \text { Score }= \begin{cases}I_{A}+I_{B}-\left(I_{A} \times I_{B}\right), & I_{A}>0 \text { and } I_{B}>0 \\
I_{A}+I_{B}+\left(I_{A} \times I_{B}\right), & I_{A}<0 \text { and } I_{B}<0\end{cases} \\
& \frac{I_{A}+I_{B}}{1-\min \left[\left|I_{A}\right|,\left|I_{B}\right|\right]} \quad \text { Otherwise }
\end{aligned}
$$

The method is derived from an expert system algorithm called Emycin [13]. The operations utilize map algebra calculation to integrate two values at a time, i.e., pair-wise calculation, while avoiding the problem of double-counting. The calculations are performed iteratively until all normalized layers are exhausted. As illustrated in Figure 6, the operation calculates the values of two attributes at a time to derive the final score, which is the integration of the values in all attributes. Through fuzzy logic operations, two QOLs (different factor contributing to QOL) can be integrated at a time until all QOLs are exhausted. Iteration 1 integrates QOL 1 and QOL2 so that only the overlapping portion of both factors values remains. By taking this portion to integrate with another QOL, QOL3, the final result is the overlapping portion among three factors; QOL1, QOL2, and QOL3.The results can be color-coded as a gradient map of integrated and locale-specific QOL in the range of $(-1,+1)$.

The nicety of the Emycin formula is that:

1. Regardless of the number of factors being used, you always "operate" on two of them in each iteration. This is called pair-wise calculation.

2. Depending on the score values of the two factors, there will be only one of the equations that is applicable. 
3. Unlike many "ordinary" algorithms, this formula allows both positive and negative contributions from factors under considerations, which is more realistic.

4. Regardless of the number of factors being considered and operated on, the resulted score will always be bounded between -1 and +1 .

5. Regardless of the sequence each factor is put into pair-wise calculation, the result is always the same.

6. Once all factors are exhausted in the calculation, one can always convert the result back to the qualitative scheme to make it more comprehensive to lay persons or people one intend to interpret the results to.

A word of caution: Both -1 and +1 are "singular" points. In other words, if you come up with a score on the contribution of a factor to be either -1 or +1 , then other factors' contributions will not matter anymore. This is not a surprise or unreasonable. Because -1 means absolutely "bad" and +1 means absolutely "good." When you have a factor that determines the quality of life to be absolutely bad, then indeed why bother to waste time to assess other factors?

To this end, one may want to adjust or shift the score from a factor that is somewhat different from the absolute values of -1 or +1 so that the pair-wise calculation may proceed logically. Again, this is not unreasonable as there is hardly anything that one can claim that is absolutely good or bad.

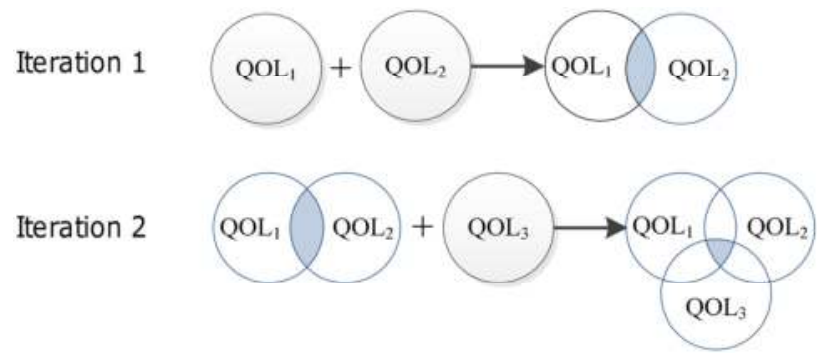

Figure 6. Illustration of pair-wise calculations.

3. Incomplete information

An additional nicety to the above approach is that one can proceed to conduct studies under incomplete information. The condition of incomplete information actually happens quite often in real life. With the kind of flexibility boasted by Emycin, you "add" the contributions from whatever data you are able to get your hand on for a city in determining its quality of life. In the case of comparing multiple cities, you may get this and that for one jurisdiction while not the same categories for all of them. By nature of the conversion, normalization, and pair-wise calculation, you would be able to derive scores on the same scheme and will be able to make comparisons.

With better understanding on the assessment framework, it is time to put such theory into real application. 


\section{Step 1: Framing the problem}

The information of interest for this case is the factors contributing to the QOL of a city/community. Relevant questions include:

1. What defines a high quality of life?: This depends on who the target audience is: elderly community, students, or married couples.

2. What factors can contribute to the QOL?:

a. Distance to: hospitals, schools, university, parks, landfill, oil wells, etc.

b. Census Data Analysis: racial mix, relative income of a population, and number of children per household

Step 2: Defining the project area

In the case of the QOL assessment, the project area was the City of College Station, Texas.

Step 3: Identify and acquiring data

Acquiring data from the City's GIS Department is crucial. From the rich collection of datasets rendered by the City [14], a number of data layers were selected for the ensuing analysis endeavor. They included census data, roads and streets, railroads, parks and green spaces, residence subdivisions, landfills, oil wells, schools, hospitals, flood plains, crime statistics, and many more.

Step 4: Extracting and preparing data

DEMs for College Station were acquired and converted into raster. This represented the base map of College Station for the following steps. Selecting only the areas pertaining to College Station attribute was also another important preparation step since we were looking at the QOL in College Station and nothing else.

\section{Step 5: Editing spatial data}

Basemap that contains areas beyond College Station were clipped off, and only the College Station boundary was left for the analysis.

Step 6: Geospatial analysis

Based on the identified QOL factors, proximities to parks, green spaces, schools, hospitals, and some other geographic features were regarded as positive contributing factors. On the other hand, closeness to such factors as landfills, oil wells, railroads, crime occurrences, and flood plains were considered to have negative impacts. The contributions of these factors, positive or negative, were mathematically formulated as distance functions from objects on corresponding data layers. In the ensuing steps, proximity maps encoded with distance functions were generated. The results from each factor layer were then combined with fuzzy logic calculation to form an integrated index between $(-1,1)$. Any number greater than 0 indicated a good QOL with anything below 0 representing bad index. The index was coded in a color scheme with a gradient from red 
to green. The color-coded QOL maps displayed clearly the patterns of QOL of the City at every specific neighborhood and locale.

To better illustrate this, four QOL factors, QOL1, QOL2, QOL3, and QOL4, were used as an example for the calculation (see Figure 7). The first fuzzy operation employed two QOLs, QOL1 and QOL2, to derive QOL12. The locales within defined proximity to QOL1 were colorcoded in green representing high QOL with the values approaching 1 . On the other hand, those in red represented low QOL with the values approaching -1. Next, QOL12 was integrated with QOL3 resulting in QOL123. The last operation was QOL123 and QOL4 as shown in the final integrated map of QOL 1234. The map results in the color gradient reflecting more green in the portion where high QOLs overlap (in the middle of the map) while the outer portion becomes more yellow to orange as a result of integrated low QOLs.

At this stage, twenty sample residential addresses were selected and tabulated in a table. By linking this table to the Address Locator tool in ArcGIS, the residential addresses were shown as a point shapefile on the map. To pinpoint the QOL of each selected address, Identify Tool was used to indicate the QOL index associated with such address.

Step 7: Generating maps and report

Map reports of this project were individually created to reflect normalized layer of each factor contributing to QOL. The normalized values (within -1 to 1 range) were shown in the legend to reflect the results from the analysis. The combined layers resulted from Emycin algorithms were exported into a group of combined layer of strictly positive factors, combined layer of strictly negative factors, and combined layer of the combination of positive and negative factors. The report concludes how QOL assessment was made possible with useful applications of GIS. Future development of applications from the QOL assessment such as the linkage to SAVEE framework was also discussed.

The QOL index illustrated in the above example sheds light on the shape of things to come with SAVEE. First of all, one may acquire the land price and/or real estate information from local authority of a jurisdiction. Using the SAVEE methodology, such information can be converted into the $(0,1)$ range for services provided by specific environments and ecosystems in an area. Similarly, the QOL index above may also be computed in the same range. Spatial statistical analyses can then be conducted to determine correlation between land prices and QOL. Useful information may be thus generated to pave way for "spatial acres of an environment or ecosystem".

\section{Concluding remarks}

The chapter depicts the natures and categories of environmental issues people are facing and how GIS can be deployed to help address them. In the context of environmental problem-solving, the systems approach for applying GIS is presented; and a few practical cases are illustrated. This organization casts a holistic view for readers to gain better comprehension of the subject matter. 


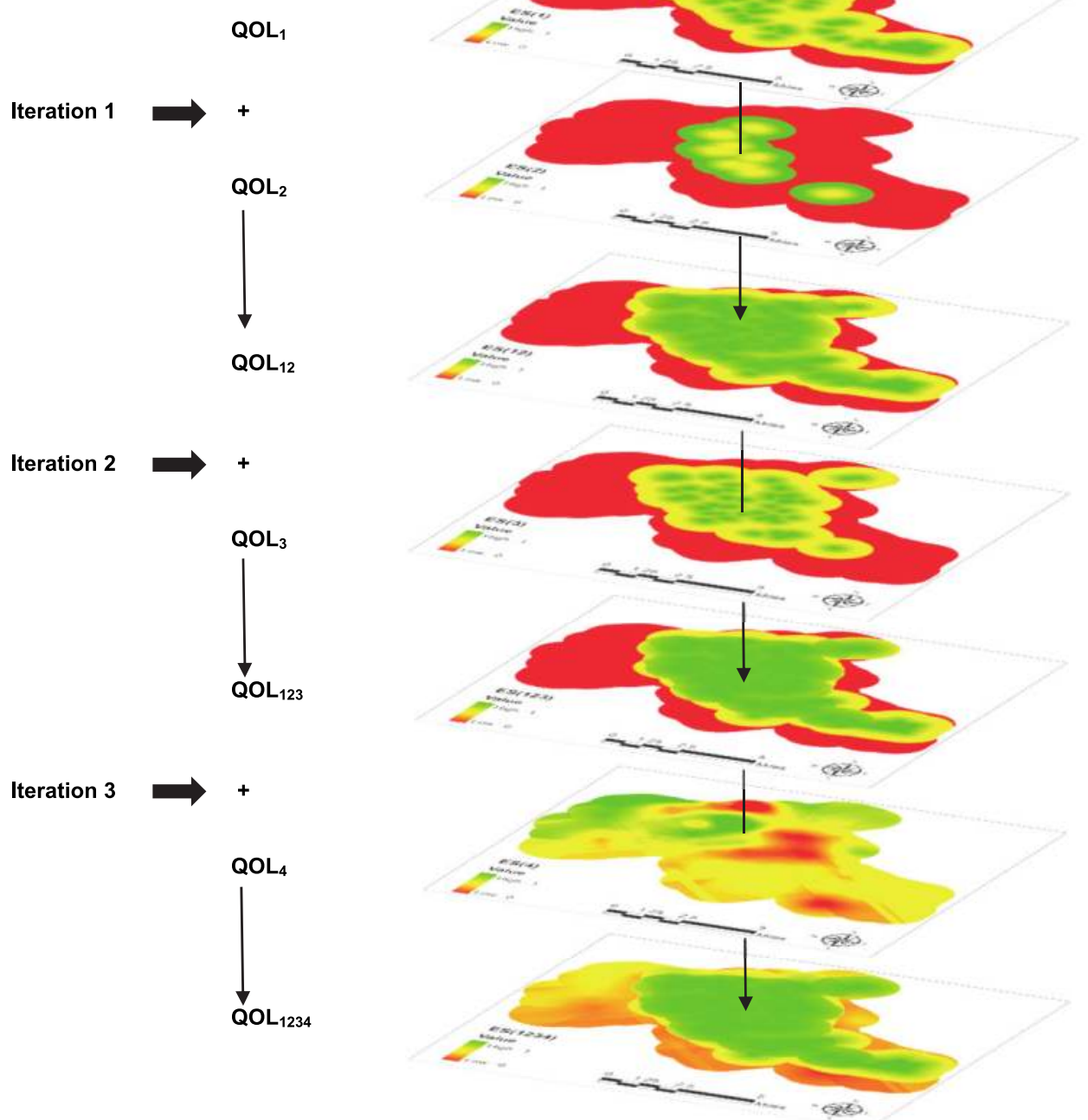

Figure 7. Iteration of map algebra to incorporate fuzzy logic to compute contributions of relevant factors to locale-specific QOL in College Station, Texas.

Problem-solving starts with shaping a mental model on to formulate a solution to the issue at hand. Steps of the solution process are then implemented through the use of appropriate data and tools enabled by GIS. Skills and knowledge to facilitate these endeavors can be best advanced by hands-on practices. For this purpose, interested readers may access the full set of documentation of learning modules at http://starr.tamu.edu/gis2012a/. The materials are from a senior course the authors teach at Texas A\&M University. It bears the same title as this chapter, "GIS for Environmental 
Problem-Solving." First conceived in the 1990s, the course has gained and maintained its popularity among the student bodies. The learning modules include well-organized stepby-step instructions of applications in ArcGIS presented in this chapter. Being offered online since 2006, this course has proven to be easy yet comprehensive for self-learning, even among students with no prior GIS background.

It is worthwhile mentioning that the approach mentioned above is for a typical GIS project for environment. There is, however, usually one step short. That is asserting monetary values associated with the environment. This issue is emerging as a priority matter in the environmental research community. For example, the monetary losses from the BP Oil Spill in the Gulf of Mexico are yet to be more plausibly determined. Taking on this issue, the authors here at the STARR LAB are developing a new research methodology called Spatial Appraisal and Valuation of Environment and Ecosystems (SAVEE). The aim of this effort is to define "spatial acre" that attaches monetary values to a geographic span of interest.

One main thrust of SAVEE is to cross-reference economic development and ecological sustainability in the framework of Sustainable Development declared in the 1992 Earth Summit and being enhanced continuously ever since. Economic development is tangible and comes with a price tag. It is plausible to assume that the intensity of development of a locale of interest can be reflected in its real estate value, which is generally available. Sampling some locales of their real estate values leads to a price list of real estate values. This price list is then converted into a uniform range between 0 and 1, a well-behaved index representing the intensity of development of locales. On the other hand, ecological sustainability of an area of interest normally does not come with a price tag. However, one may systematically incorporate pertinent ecological services it renders into consideration and develop an index that has the same range of $(0,1)$. The numbers approaching 1 represent higher sustainability, and the opposites represent lower indices. On the basis of equitability between development and environment, the two index systems may then be mapped. The mapping leads to assigning monetary values associated with development sites to ecological locales with comparable index numbers.

Learning is a life-long process; so are the advances of knowledge and technologies. On the environmental GIS front, asserting monetary values to a system under study has become an imperative. The authors are hopeful that the general framework stipulated in SAVEE shall be advanced to explore this new territory. Only labeling it with dollar signs would make stakeholders appreciate more of our environment of its values. After all, without such dollar values, it is difficult for stakeholders and authorities to understand the magnitude of the environmental problems at hand. It is contended that SAVEE and other similar effort will make a significant contribution to environmental sectors in general and the advancement of GIS.

\section{Author details}

Koushen Douglas Loh and Sasathorn Tapaneeyakul Department of Ecosystem Science and Management, Texas AEM University, USA 


\section{References}

[1] Dueker K.J, Kjerne D (1989) Multipurpose Cadastre: Terms and Definitions. Falls Church: American Society for Photogrammetry and Remote Sensing. 12 p.

[2] Hein L, Van Koppen K, De Groot R.S, Van Ierland E.C (2006) Spatial Scales, Stakeholders and the Valuation of Ecosystem Services. J Ecol econ. 57(2): 209-228.

[3] West Nile Virus Activity in Brazos County [Internet]. College Station (TX): AgriLIFE Extension; Agricultural and Environmental Safety; 2012 [cited 2012 April 21]. Available from: http://www-aes.tamu.edu/public-health-vector-and-mosquito-control/brazoscounty-mosquito-borne-disease-surveillance/west-nile-virus-activity-in-brazos-county/

[4] Maling D.H (1991) Coordinate Systems and Map Projections for GIS. In: Maguire D.J, Goodchild M.F, Rhind D.W, editors. Geographical Information Systems: Principles and Applications. London: Longman Group UK. Vol. 1, pp. 135-146.

[5] Knabb R.D, Rhome J.R, Brown D.P (2006) Tropical Cyclone Report: Hurricane Katrina: 23-30 August 2005. National Hurricane Center.

[6] Jonkman S.N, Maaskant B, Boyd E, Levitan M.L (2009) Loss of Life Caused by the Flooding of New Orleans after Hurricane Katrina: Analysis of the Relationship between Flood Characteristics and Mortality. Risk anal. j. 29(5): 676-698.

[7] Atlas: The Louisiana Statewide GIS [Internet]. Baton Rouge (LA): Louisiana State University CADGIS Research Laboratory; 2009 [cited 2012 April 23]. Available from: http://atlas.lsu.edu/

[8] ArcGIS Desktop 9.3 Help [Internet]. Redland (CA): Environmental Systems Research Institute; 2008 [cited 2012 April 23]. Available from:

http://webhelp.esri.com/arcgisdesktop/9.3/index.cfm?TopicName=Sample_VBA_code

[9] Atlas of Canada Quality of Life [Internet]. [Place unknown]: National Resources Canada; 2009 [cited 2012 April 23]. Available from:

http://atlas.nrcan.gc.ca/auth/english/maps/peopleandsociety/QOL

[10] The 2011 Utah Foundation Quality of Life Index: First Biennial Survey Reveals Strengths, Weaknesses [Internet]. Salt Lake City (UT): Utah Foundation; 2011[cited 2012 April 27]. Available from: http://www.utahfoundation.org/img/pdfs/rr703.pdf

[11] City and County of San Francisco as Successor to the Redevelopment Agency [Internet]. San Francisco: San Francisco Redevelopment Agency; 2012 [cited 2012 April 27]. Available from: http://www.sfredevelopment.org/

[12] The Quality of Life Foundation [Internet]. San Francisco: The Quality of Life Foundation; [Date unknown] [cited 2012 April 27]. Available from:

http://www.qualityoflifefoundation.org/

[13] van Melle W, Shortliffe E.H, Buchanan B.G (1984) EMYCIN: A Knowledge Engineer's Tool for Constructing Rule-Based Expert Systems. In: Shortliffe, E.H., editor. Rule-Based Expert Systems: The MYCIN Experiments of the Stanford Heuristic Programming Project. Reading (MA): Addison-Wesley. pp. 302-313.

[14] GIS - Geographic Information Services [Internet]. College Station (TX): City of College Station GIS; 2001 [cited 2012 April 29]. Available from:

http://www.cstx.gov/index.aspx?page=3683 